\title{
Lin28a ameliorates glucotoxicity-induced $\beta$-cell dysfunction and apoptosis
}

\author{
Yeo Jin Hwang ${ }^{1}$, Gwon-Soo Jung ${ }^{2}$, WonBae Jeon ${ }^{3}$ E Kyeong-Min Lee ${ }^{3, *}$ \\ ${ }^{1}$ Division of Electronics \& Information System, Daegu Gyeongbuk Institute of Science and Technology, Daegu 42988, ${ }^{2} \mathrm{New}$ Drug \\ Development Center, Daegu-Gyeongbuk Medical Innovation Foundation, Daegu 41061, ${ }^{3}$ Division of Biotechnology, Daegu Gyeongbuk \\ Institute of Science and Technology, Daegu 42988, Korea
}

An excessive and prolonged increase in glucose levels causes $\beta$-cell dysregulation, which is accompanied by impaired insulin synthesis and secretion, a condition known as glucotoxicity. Although it is known that both Lin28a and Lin28b regulate glucose metabolism, other molecular mechanisms that may protect against glucotoxicity are poorly understood. We investigated whether Lin28a overexpression can improve glucotoxicityinduced $\beta$-cell dysregulation in INS-1 and primary rat islet cells. INS-1, a rat insulinoma cell line was cultured and primary rat islet cells were isolated from SD-rats. To define the effect of Lin28a in chronic high glucose-induced $\beta$-cell dysregulation, we performed several in vitro and ex-vivo experiments. Chronic exposure to high glucose led to a downregulation of Lin28a mRNA and protein expression, followed by a decrease in insulin mRNA expression and secretion in $\beta$-cells. The mRNA and protein expression levels of PDX-1 and BETA2, were reduced; The levels of apoptotic factors, including c-caspase 3 and the $\mathrm{Bax} / \mathrm{BCl}-2$ ratio, were increased due to glucotoxicity. Adenovirusmediated Lin28a overexpression in $\beta$-cells reversed the glucotoxicity-induced reduction of insulin secretion and insulin mRNA expression via regulation of $\beta$-cell-enriched transcription factors such as PDX-1 and BETA2. Adenovirus-mediated overexpression of Lin28a downregulated the glucotoxicity-induced upregulation of c-caspase 3 levels and the Bax/Bcl-2 ratio, while inhibition of endogenous Lin28a by small interfering RNA resulted in their up-regulation. Lin28a counteracted glucotoxicity-induced downregulation of $p$-Akt and p-mTOR. Our results suggest that Lin28a protects pancreatic $\beta$-cells from glucotoxicity through inhibition of apoptotic factors via the PI3 kinase/Akt/mTOR pathway. [BMB Reports 2021; 54(4): 215-220]

${ }^{*}$ Corresponding author. Tel: +82-53-785-2430; Fax: +82-53-785-2439; E-mail: leekm1009@dgist.ac.kr

https://doi.org/10.5483/BMBRep.2021.54.4.255

Received 13 November 2020, Revised 16 December 2020, Accepted 9 March 2021

Keywords: Apoptosis, Glucotoxicity, Insulin, Lin28a, mTOR, PI3K/Akt

\section{INTRODUCTION}

Prolonged exposure to hyperglycemia induces $\beta$-cell dysregulation and ultimately cell death; phenomenon is known as glucotoxicity (1-5). In type 2 diabetes, insulin secretion progressively increases in an attempt to regulate glucose homeostasis during insulin resistance (6). However, chronic up-regulation of insulin secretion causes defects in insulin secretion, leading to constant hyperglycemia (7). Chronic hyperglycemia is toxic to pancreatic $\beta$-cells, impairing insulin synthesis and secretion, and inducing apoptosis, as has been observed in type 2 diabetes $(4,8)$.

In type 2 diabetes, the chronic toxicity of high glucose, oxidative stress and free fatty acids is involved in the increase of pro-apoptotic factors, such as caspases, and decreased antiapoptotic factors, such as $\mathrm{BCl}-2$ in $\beta$-cells $(5,9)$. In several studies, glucotoxicity-induced $\beta$-cell apoptosis has been shown to be related to an imbalance between pro-apoptotic proteins and anti-apoptotic proteins, such as the $\mathrm{Bax} / \mathrm{Bcl}-2$ ratio, which was skewed towards apoptosis in insulinoma cell culture and isolated islets in vitro (10). Apoptosis in $\beta$-cells is stimulated by prolonged elevated glucose through caspase3 activation (11).

Lin28a is an RNA-binding protein that selectively represses the expression of microRNAs that regulate glucose metabolism as well as cell proliferation and the differentiation of embryonic cells, stem cells and cancer (12-16). Lin28 consists of two homologues, Lin28a and Lin28b, with similar structural and functional properties, but which have a few differences (17). Recently, it was reported that Lin28a regulates the miRNA Let-7 which acts as a suppressor of genes related to the insulin signaling pathway (15). In Lin28a transgenic mice, Lin28a improved an insulin-sensitive state and prevented the development of diabetes, while Let-7 led to insulin resistance and an impairment of glucose tolerance in the skeletal muscle and brown adipose tissue of Let-7 transgenic mice (15). Impairment of insulin secretion and glucose intolerance were observed in mice with whole body and pancreas-specific overexpression of Let-7 (18). Although these observations suggest Lin28a has an important role in regulating glucose homeostasis, the question of whether Lin28a reduces glucotoxicity-induced $\beta$-cell dysfunction and apoptosis has yet to be considered.

ISSN: 1976-670X (electronic edition)

Copyright (c) 2021 by the The Korean Society for Biochemistry and Molecular Biology

(c) This is an open-access article distributed under the terms of the Creative Commons Attribution Non-Commercial License (http://creativecommons.org/licenses/by-nc/4.0) which permits unrestricted non-commercial use, distribution, and reproduction in any medium, provided the original work is properly cited. 
We investigated whether glucotoxicity affects the expression in $\beta$-cells of Lin28a, which is associated with apoptosis. We also determined the role of Lin28a in glucotoxicity-induced $\beta$-cell dysfunction and apoptosis and investigated its mechanism of action.

\section{RESULTS}

\section{Glucotoxicity induced $\beta$-cell dysfunction, apoptosis, and Lin28a downregulation in primary rat islets}

Glucotoxicity is known to have harmful effects on $\beta$-cells causing dysfunction and ultimately leading to $\beta$-cell death (1-3, 5). We examined whether the level of Lin28a in pancreatic $\beta$-cells was altered by glucotoxicity. To confirm the induction of glucotoxicity in $\beta$-cells, the expression of insulin and its transcription factors was measured. When primary rat islet cells were cultured in high glucose (30 mML D-glucose) for $48 \mathrm{~h}$, the gene levels of insulin-1 and insulin-2 decreased over time (Fig. 1C, D). Incubation with high glucose for $48 \mathrm{~h}$ also impaired glucose-stimulated insulin secretion (GSIS) in rat islet cells (Fig. 1E). The mRNA and protein expressions of PDX-1 and BETA2 were reduced by high glucose levels in rat islet cells (Fig. 1 F-H). However, Bax, cleaved-caspase3 (c-caspase3),
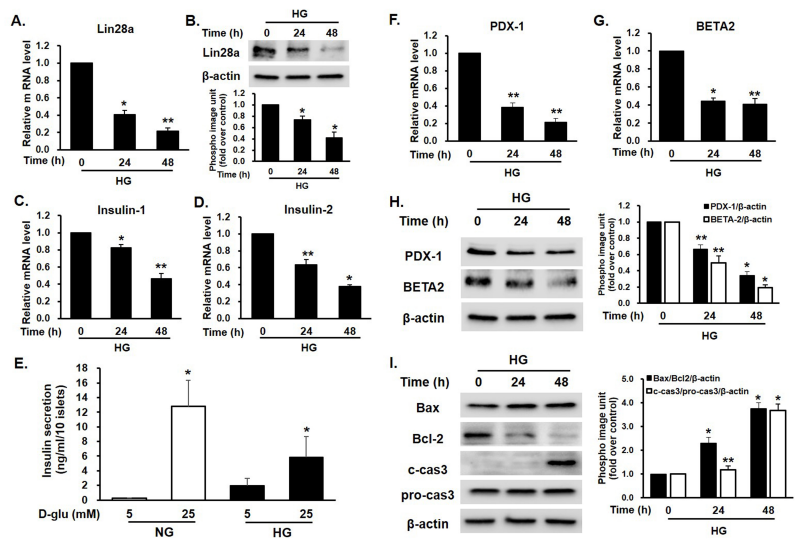

Fig. 1. Effect of glucotoxicity on Lin28a expression, $\beta$-cell function and apoptosis in primary rat islet cells. Rat islet cells were cultured in high glucose ( $\mathrm{HG}, 30 \mathrm{mM}$ D-glucose) for the time shown. (A, C, D, F, G) RT-qPCR analysis for Lin28a, insulin-1, insulin-2, PDX-1, and BETA 2 in high glucose-incubated rat islets cells. Data are from three independent experiments and values are shown as the mean + SEM $* P$ value $<0.001$ and $* * P$ value $<0.01$ vs. $0 \mathrm{~h}$. $(\mathrm{B}, \mathrm{H}, \mathrm{I})$ Western blot analysis for Lin28a, PDX-1, BETA2, Bax, Bcl-2, c-caspase3 (c-cas3) and pro-caspase3 (pro-cas3) in high glucose-incubated rat islet cells. Data are from three independent measurements and values are shown as the mean \pm SEM. $* P<0.01$, $* * \mathrm{P}<0.05$ vs. 0 h. (E) Quantification of glucose-stimulated insulin secretion (GSIS) of rat islet cells incubated with high glucose containing medium for $48 \mathrm{~h}$. Data are from three independent experiments and values are shown as the mean $\pm \mathrm{SEM}$. $* \mathrm{P}$ value $<$ 0.05 vs. $5 \mathrm{mM}$ D-glucose. PDX-1, pancreatic and duodenal homeobox 1; BETA2, basic helix-loop-helix type of transcription factor; GSIS, glucosestimulated insulin secretion. and the $\mathrm{Bax} / \mathrm{Bcl}-2$ ratio, all increased in response to high glucose in rat islet cells. Bcl-2 protein expression was decreased by high levels of glucose in rat islet cells (Fig. 1I). The mRNA and protein expression of Lin28a were reduced by exposure to high glucose in rat islet cells (Fig. 1A, B). These results suggest that glucotoxicity induces downregulation of Lin28a expression as well as $\beta$-cell dysfunction and apoptosis.

\section{Overexpression of Lin28a ameliorated glucotoxicity-stimulated $\beta$-cell dysfunction}

We tested whether the overexpression of Lin28a ameliorated glucotoxicity-stimulated $\beta$-cell dysfunction in primary rat islet cells. As shown in Fig. 2A, B, adenovirus-mediated overexpression of Lin28a in rat islets ameliorated the downregulation of insulin mRNA induced by high glucose. High glucose impaired GSIS; however, Ad-Lin28a ameliorated high glucose-induced impairment of GSIS (Fig. 2C). Ad-Lin28a restored high glucoseinduced downregulation of mRNA (Fig. 2D) and protein (Fig. 2E) levels of PDX-1 and BETA2 in INS-1 cells. These results indicate that Lin28a decreased $\beta$-cell dysfunction via regulation of $\beta$-cell-enriched transcription factors.
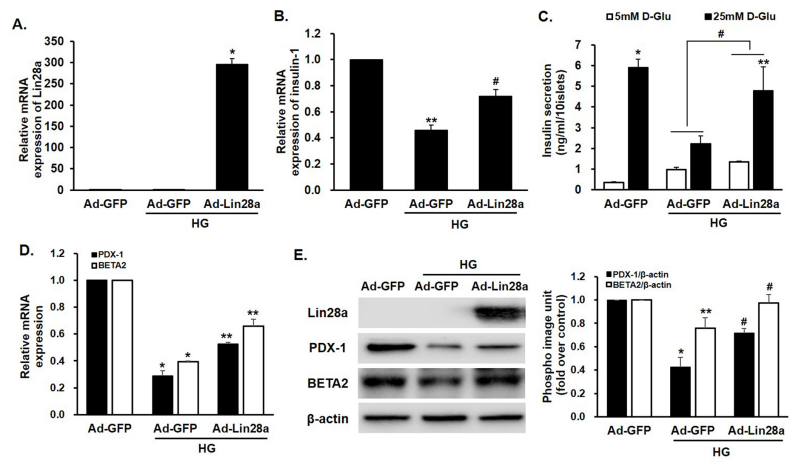

Fig. 2. Effect of Lin28a overexpression on glucotoxicity-induced $\beta$-cell dysfunction. Rat islet cells or INS-1 cells were incubated with Ad-Lin28a or Ad-GFP at a MOI of 20 and then cultured in normal glucose (NG, $5 \mathrm{mM}$ D-glucose) or high glucose (HG, $30 \mathrm{mM}$ Dglucose) for 48h. (A and B) RT-qPCR for Lin28a and insulin-1 in rat islet cells. Data are from three independent experiments and values are shown as the mean + SEM. $* P$ value $<0.001$ and $* * P$ value $<0.01$ vs. NG/Ad-GFP. ${ }^{\#}$ value $<0.05$ vs. HG/Ad-GFP. (C) Quantification of GSIS of rat islets. Data are from three independent experiments and values are shown as the mean \pm SEM. ${ }^{* P}$ value $<$ 0.01 and $* * P$ value $<0.05$ vs. $5 \mathrm{mM}$ D-glucose. ${ }^{\#} \mathrm{P}$ value $<0.05$ vs. HG/Ad-GFP. (D) RT-qPCR for PDX-1 and BETA 2 in INS-1 cells. Data are from three independent experiments and values are shown as the mean \pm SEM. ${ }^{*} P$ value $<0.001$ vs. NG/Ad-GFP. $* * P$ value $<0.01$ vs. HG/Ad-GFP. (E) Western blot analysis for Lin28a, PDX-1and BETA2 in INS-1 cells. Data are from three independent experiments and values are shown as the mean \pm SEM. $* \mathrm{P}$ value $<$ 0.01 and $* * P$ value $<0.05$ vs. NG/Ad-GFP. " $P$ value $<0.05$ vs. HG/Ad-GFP. PDX-1, pancreatic and duodenal homeobox 1; BETA2, basic helix-loop-helix type of transcription factor; GSIS, glucosestimulated insulin secretion. 


\section{Lin28a regulated apoptotic factors in pancreatic $\beta$-cells} We investigated whether Lin28a inhibits glucotoxicity-stimulated apoptosis in primary rat islet cells. As shown in Fig. $3 \mathrm{~A}$ and Supplementary Fig. 1, Lin28a overexpression in rat islet cells caused by infection with Ad-Lin28a inhibited the high glucoseinduced the mRNA and protein levels of Bax/Bcl-2 ratio induced by high glucose. Lin28a overexpression decreased the expression of c-caspase3 protein induced by high glucose (Fig. 3A). To investigate the impact of Lin28a overexpression on the survival of $\beta$-cells, we stained islets to detect dead cells using Ethidium-1 to stain dead cells. Dead cells stained red were increased in high glucose and Ad-GFP treated $\beta$-cells. The number of dead cells induced by high glucose were decreased when Lin28a was overexpressed (Fig. 3B). To investigate whether Lin28a regulates the apoptotic factors in $\beta$-cell, INS-1 cells were transfected with siLin28a. The protein levels of Bax and c-caspase 3 were increased, while the Bcl-2 protein levels were decreased by Lin28a inhibition. The ratio of $\mathrm{Bax} / \mathrm{Bcl}-2$ was significantly up-regulated by siRNA administration (Fig. 3C). These results indicate that Lin28a regulates the expression of apoptotic factors.

\section{Lin28a inhibited glucotoxicity-stimulated apoptosis via the Akt pathway}

The role of $\mathrm{PI} 3$ kinase/Akt/mTOR (PI3K/Akt/mTOR) axis in $\beta$-cell growth, survival, and function has been studied in detail $(19,20)$. We examined whether the protective effects of Lin28
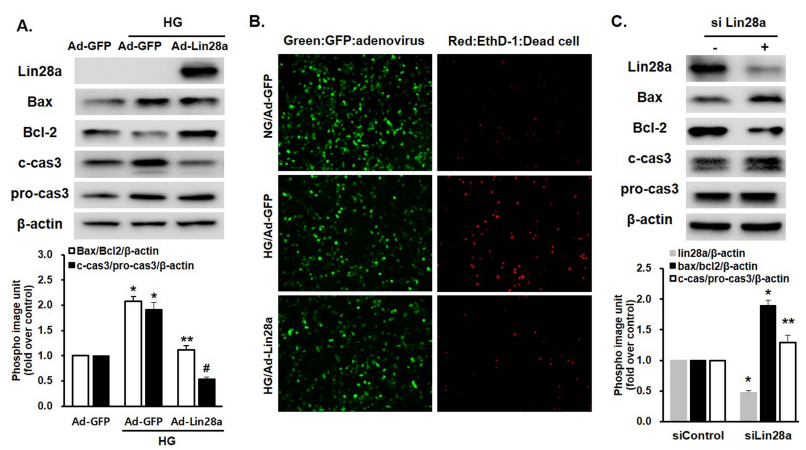

Fig. 3. Effect of Lin28a on apoptotic factors in pancreatic $\beta$-cells. (A, B) Rat islet cells were incubated with Ad-Lin28a or Ad-GFP at a $\mathrm{MOI}$ of 20 and then cultured in normal glucose (NG, $5 \mathrm{mM}$ D-glucose) or high glucose (HG, $30 \mathrm{mM}$ D-glucose) for 48h. (A) Western blot analysis for Lin28a, Bax, Bcl-2, c-caspase3 (c-cas3) and pro-caspase3 (pro-cas3) in INS-1 cells. Quantification of western blot results are from three independent experiments (bottom panel) and values are expressed as the mean + SEM. $* P$ value $<$ 0.01 vs. NG/Ad-GFP. ${ }^{*} P$ value $<0.01$ and ${ }^{\#} \mathrm{P}$ value $<0.05$ vs. HG/Ad-GFP. (B) Dead cell staining with EthD-1 in INS-1 cells. (Magnification, $\times 200$ ) (C) INS-1 cells were transfected with siRNAcontrol (siControl) or siRNA-Lin28a (siLin28a) and then cultured for $24 \mathrm{~h}$. Quantification of western blot results are from three independent experiments (bottom panel) and values are expressed as the mean \pm SEM. ${ }^{* P}$ value $<0.001$ and $* * P$ value $<0.05$ vs. siControl. in glucotoxicity-stimulated apoptosis were related to the PI3K/ Akt/mTOR pathway. Treatment with high levels of glucose decreased the phosphorylation of Akt and mTOR in INS-1 cells. Adenovirus-mediated overexpression of Lin28a effectively recovered the decreased phosphorylation of Akt and mTOR caused by high glucose (Fig. 4, left). However, the levels of Akt and mTOR phosphorylation following the administration of Wortmannin, a specific inhibitor of PI3K, treated cells, were not decreased by high glucose and were not changed by Lin28a overexpression (Fig. 4, right). These results indicated that Lin28a regulated glucotoxicity-related genes via the PI3K/ $\mathrm{Akt} / \mathrm{mTOR}$ pathway in $\beta$-cells.

\section{DISCUSSION}

In this study, we established that chronic high glucose induced the downregulation of Lin28a expression, resulting in decreases in insulin mRNA levels and secretion, as well as increases in the $\mathrm{Bax} / \mathrm{Bcl}-2$ ratio and the levels of c-caspase 3 proteins. The adenovirus-mediated overexpression of Lin28a ameliorated glucotoxicity-induced $\beta$-cell dysfunction. Ad-Lin28a inhibited the glucotoxicity-induced apoptotic factors, such as c-caspase3, and the $\mathrm{Bax} / \mathrm{BCl}-2$ ratio, while inhibition of Lin28a, by transfection with siRNA-Lin28a, increased these apoptotic factors. Ad-Lin28a recovered the glucotoxicity-induced downregulation of Akt and mTOR phosphorylation. These observations indicate that Lin28a ameliorates glucotoxicity-stimulated $\beta$-cell dysfunction and apoptosis via regulation of the PI3K/Akt/mTOR pathway as well as $\beta$-cell enriched transcription factors.

Recently, Lin28a was shown to regulate glucose metabolism

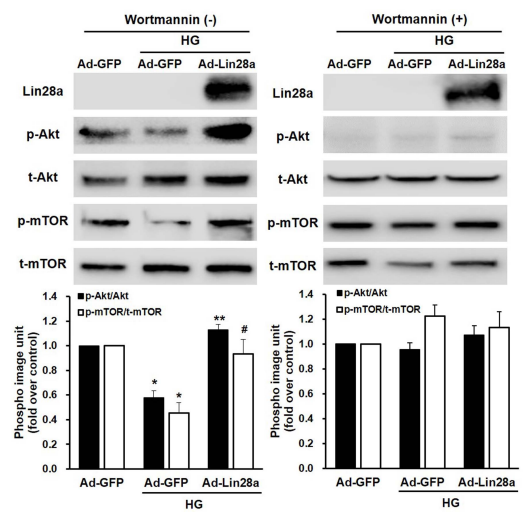

Fig. 4. Effect of Lin28a overexpression on the $\mathrm{PI} 3 \mathrm{~K} / \mathrm{Akt} / \mathrm{mTOR}$ pathway. INS-1 cells were incubated with Ad-Lin28a or Ad-GFP at a MOI of 20 and then incubated with high glucose (HG, 30 $\mathrm{mM}$ D-glucose) for $48 \mathrm{~h}$. Cells were treated with wortmannin, PI3K inhibitor, for $1 \mathrm{~h}$ before harvest (right panel). Western blot analysis for Lin28a, p-Akt, t-Akt, p-mTOR and t-mTOR. Quantification of western blots are from three independent experiments (bottom panel) and values are expressed as the mean \pm SEM. ${ }^{* P}$ value $<0.01$ vs. NG/Ad-GFP. ${ }^{* * P}$ value $<0.001$ and ${ }^{\#} \mathrm{P}$ value $<$ 0.01 vs. HG/Ad-GFP. 
and insulin sensitivity (15). Hypothalamic ventromedial nucleus specific overexpression of Lin28a induced a significant amelioration in glucose metabolism, while downregulation of Lin28a due to a high fat diet was detrimental (21). Chronic increase of glucose contributed to $\beta$-cell dysfunction and apoptosis in type 2 diabetes, as it inhibits insulin gene expression and elevates apoptotic factors (2-4). We demonstrated that Lin28a plays a critical role in the glucotoxicity induced by a prolonged high level of glucose in $\beta$-cells. In this study, we demonstrate that, under glucotoxicity conditions, the gene and protein expressions of Lin28a were significantly reduced in primary rat islet cells, while $\beta$-cell dysfunction and the expressions of apoptotic factors were increased.

Lin28a overexpression inhibited high glucose-induced downregulation of the expression of the insulin gene and GSIS in rat islet cells. PDX-1 and BETA2/NeuroD play critical roles in regulating insulin gene expression as pancreatic $\beta$-cell specific transcription factors (22). Lin28a overexpression recovered the high glucose-induced reduction of PDX-1 and BETA2. These data strongly suggest that Lin28a ameliorates glucotoxicityinduced $\beta$-cell dysfunction via the regulation of PDX- 1 and BETA2 expression.

The up-regulation of pro-apoptotic factors, such as c-caspase 3 activates the apoptotic process $(4,23)$. The $\mathrm{BCl}-2$ family is a key mediator of the process of apoptosis. The relative ratio of pro-apoptotic factors and anti-apoptotic factors, such as the $\mathrm{Bax} / \mathrm{Bcl}-2$ ratio, plays an important role in determination of cell survival or death $(11,24)$. An increase in the Bax/Bcl-2 ratio has found to be associated with a high risk of apoptotic activation $(25,26)$. Our data indicates that overexpression of Lin28a inhibited the levels of Bax and c-caspase3 induced by high levels of glucose, decreasing the $\mathrm{Bax} / \mathrm{Bcl}-2$ ratio in rat islet cells. When endogenous Lin28a expression in INS-1 cells was inhibited by Lin28a-specific siRNA, Bcl-2 expression was decreased while the levels of Bax and c-caspase3 were increased, resulting in an increase of the $\mathrm{Bax} / \mathrm{Bcl}-2$ ratio. Collectively, these data suggest that Lin28a can modulate anti-apoptotic and pro-apoptotic factors, and possibly improve the $\beta$-cell dysregulation that results from metabolic stress caused by chronically elevated glucose levels.

Several studies have shown that constitutively active Akt inhibits fatty acid-stimulated apoptosis in $\beta$-cells $(27,28)$, and $\beta$-cell specific overexpression of Akt has also been shown to prolong cell survival and protect against streptozotocin-induced diabetes (29). In several cells, pancreatic $\beta$-cells, muscle cell and cardiomyocytes Lin28a has been reported to prevent apoptosis and regulate the $\mathrm{PI} 3 \mathrm{~K} / \mathrm{Akt} / \mathrm{mTOR}$ pathway through downregulation of micro RNA Let-7 $(15,30,31)$. We found that INS-1 cells treated with high glucose had decreased phosphorylation of Akt and mTOR while Lin28a overexpression recovered the high glucose-induced downregulation of p-Akt and p-mTOR. These data suggest that Lin28a regulates glucotoxicity-induced downregulation of the PI3K/Akt/mTOR pathway in $\beta$-cells.
It has been reported that high glucose suppresses the expression of the insulin gene, and its transcription factors such as PDX-1 and BETA 2, and insulin secretion independently of apoptosis $(3,10)$. Our data showed that Lin28a expression in $\beta$-cells was decreased by glucotoxicity and previous reports determined the suppression of Lin28a expression in cardiomyocytes by diabetes or ischemic insults (31). However, we have not identified the relationship between of high glucoseinduced apoptosis and the high glucose-related regulatory mechanisms of Lin28a expression, and further research is expected in the future.

In summary, the present study shows that a chronic increase of glucose induces $\beta$-cell dysfunction and apoptosis, resulting in the downregulation of Lin28a expression. Under glucotoxic conditions, Lin28a up-regulates the expression and secretion of the insulin gene, while decreasing the $\mathrm{Bax} / \mathrm{Bcl}-2$ ratio and c-caspase3 protein expression via the $\mathrm{PI} 3 \mathrm{~K} / \mathrm{Akt} / \mathrm{mTOR}$ pathway. These results suggest that Lin28a has a crucial role in antiapoptosis, making it a promising therapeutic target for addressing pancreatic $\beta$-cell dysfunction caused by glucotoxicity.

\section{MATERIALS AND METHODS}

\section{Cell culture}

The INS-1 rat insulinoma cell line was cultured as previously described (22). To determine the effect of high glucose, cells were treated with $11.2 \mathrm{mM}$ or $30 \mathrm{mM}$ D-glucose for the indicated time. To investigate the effect of overexpression of Lin28a, cells were infected with Ad-Lin28a for $2 \mathrm{~h}$ in serumfree medium, and then incubated with fresh medium containing $11.2 \mathrm{mM}$ or $30 \mathrm{mM}$ D-glucose for $48 \mathrm{~h}$.

\section{Preparation of primary rat islets}

Primary rat islets were isolated by collagenase digestion, and cultured as described previously (22). We performed all animal experiments in accordance with national guidelines and applicable national laws regarding animal protection. DGIST's Animal Care and Use Committee approved the animal protocols (DGIST-IACUC-19052103-03). The islets were treated with AdLin28a for $2 \mathrm{~h}$ in serum free medium, and then incubated with fresh medium containing $11.2 \mathrm{mM}$ or $30 \mathrm{mM} \mathrm{D-glucose}$ for $48 \mathrm{~h}$.

\section{Preparation of recombinant adenovirus}

Recombinant adenovirus was prepared in a previously reported method (32).

\section{RNA expression analysis by real time-qPCR}

RNA was obtained from conditioned cells using RNA Extraction Kits (Takara, Japan). cDNAs were generated using Reverse Transcription Kits (Applied Biosystems, USA). Gene expression levels were evaluated using Applied Biosystems SYBR Green PCR master mix kit in an ABI 7500 qPCR System. The qPCR conditions and the primers designed by Primer Express Software ver. 3.0 are described in Supplementary Table 1. 


\section{Western blotting analysis}

The cells or islets were lysed using RIPA buffer (Sigma, USA) with protease inhibitor and phosphatase inhibitor, and the cell lysate was harvested by centrifugation at $13000 \mathrm{rpm}$ for 20 min. The protein samples were separated by Mini-PROTEAN ${ }^{\mathbb{R}}$ $\mathrm{TGX}^{\mathrm{TM}}$ Gels (Bio-Rad, USA) and electrophoretically transferred onto membranes. The membranes were incubated with blocking solution containing $5 \%$ BSA (Sigma) for $1 \mathrm{~h}$, and further incubated with anti-Lin28a antibody, anti-Bax antibody, anti-cleavedcaspase3 antibody, anti-pro-caspase3 antibody, anti-phospho-Akt antibody, anti-Akt antibody, anti-phospho-mTOR antibody and anti-mTOR antibody (Cell Signaling, USA), anti-Bcl-2 antibody and anti-PDX-1 antibody (Santacruz, USA), or anti-BETA2 antibody (Abcam, UK). Antibodies were detected using a horseradish peroxidase-conjugated secondary antibody (Santacruz) and Clarity Max ${ }^{\mathrm{TM}}$ Western ECL Substrate (Bio-Rad). The membranes were re-blotted with an anti-actin antibody (Santacruz) and immunoblot images were analyzed by ChemiDoc ${ }^{\mathrm{TM}} \mathrm{XRS}+$ (Bio-Rad), and the intensity of the bands was quantified using Image Lab ${ }^{\text {TM }}$ Software (Bio-Rad).

\section{Dead cell staining}

Dead cell staining was performed using LIVE/DEAD Viability/ Cytotoxicity Assay Kit (Invitrogen, USA) according to the manufacturer's protocol, with some modifications. INS-1 cells were incubated for 2 days in $36 \mathrm{~mm}$ dishes and then washed twice with Dulbecco's phosphate-buffered saline. To stain dead cells, the cells were incubated with $4 \mu \mathrm{M}$ EthD-1 solution for $10 \mathrm{~min}$. Fluorescein labeling images were obtained at wavelengths ranging from $\sim 495 \mathrm{~nm} / \sim 635 \mathrm{~nm}$ (Red:dead cell) using a fluorescence microscope (Leica, Germany) and a MataVue Imaging System Version 7.8.0.0 (Molecular Devices, USA).

\section{Construction of siRNA for Lin28a}

Predesigned siRNAs for the control and Lin28a were purchased from BIONEER (South Korea). The Lin28a and control siRNA (C-siRNA) sequences are as follows: rat siLin28a, GAC GUC UUU GUG CAC CAG A tt; C-siRNA, CCU ACG CCA CCA AUU UGG U tt. $100 \mathrm{nM}$ siRNA was transfected into INS-1 cells using transfection reagent (Lipofectamine 2000, Invitrogen, USA) in transfection media (Opti-MEM, Gibco, USA) for $4 \mathrm{~h}$; then they were moved to a culture medium containing $2 \%$ FBS and collected $\sim 24 \mathrm{~h}$ after transfection. The effect of Lin28a siRNA was confirmed by western blot analysis.

\section{Glucose-stimulated insulin secretion (GSIS)}

Ten islets (triplets/condition) were infected with adenovirus Lin28a with a MOI of 20 in serum-free medium; adenovirus GFP was used for the control group. After $2 \mathrm{~h}$, the islets were transferred to fresh medium with $2 \% \mathrm{FBS}$ and $5 \mathrm{mM}$ or $30 \mathrm{mM}$ D-glucose for $48 \mathrm{~h}$. The islets were then starved in $2 \%$ FBS and $5 \mathrm{mM} \mathrm{D-glucose}$ medium for $5 \mathrm{~h}$ and washed with $5 \mathrm{mM}$ D-glucose KRBB. The islets were then replaced with a $5 \mathrm{mM}$ or $25 \mathrm{mM} \mathrm{KRBB}$ and incubated for $1 \mathrm{~h}$. The supernatant was gently collected and stored at $-72^{\circ} \mathrm{C}$ until measurement. Changes in insulin secretion were analyzed using Rat Ultrasensitive Insulin ELISA (ALPCO, USA).

\section{Statistical analysis}

Data were expressed as the mean \pm SEM. ANOVA was used to evaluate the variance, followed by a post- hoc least significant difference test. Statistical significance is indicated by $\mathrm{P}$ values $<0.05$.

\section{ACKNOWLEDGEMENTS}

This work was supported by DGIST projects 21-BT-06, NRF2021R1A2C1011314 and NRF-2018R1C1B6008955 from the National Research Foundation of Korea funded by the Ministry of Science, ICT of the Republic of Korea.

\section{CONFLICTS OF INTEREST}

The authors have no conflicting interests.

\section{REFERENCES}

1. Korsgren O, Jansson L, Sandler S et al (1990) Hyperglycemiainduced $\mathrm{B}$ cell toxicity. The fate of pancreatic islets transplanted into diabetic mice is dependent on their genetic background. J Clin Invest 86, 2161-2168

2. Unger RH and Grundy S (1985) Hyperglycaemia as an inducer as well as a consequence of impaired islet cell function and insulin resistance: implications for the management of diabetes. Diabetologia 28, 119-121

3. Marshak S, Leibowitz G, Bertuzzi F et al (1999) Impaired beta-cell functions induced by chronic exposure of cultured human pancreatic islets to high glucose. Diabetes 48, 1230-1236

4. Donath MY, Gross DJ, Cerasi E et al (1999) Hyperglycemiainduced beta-cell apoptosis in pancreatic islets of Psammomys obesus during development of diabetes. Diabetes 48, 738744

5. Poitout V and Robertson RP (2008) Glucolipotoxicity: fuel excess and $\beta$-cell dysfunction. Endocr Rev 29, 351-366

6. Kahn SE, Hull RL and Utzschneider KM (2006) Mechanisms linking obesity to insulin resistance and type 2 diabetes. Nature 444, 840-846

7. Bonner-Weir S, Trent DF and Weir GC (1983) Partial pancreatectomy in the rat and subsequent defect in glucose-induced insulin release. J Clin Invest 71, 1544-1553

8. Del Prato S (2009) Role of glucotoxicity and lipotoxicity in the pathophysiology of type 2 diabetes mellitus and emerging treatment strategies. Diabetic Med 26, 1185-1192

9. Mandrup-Poulsen T (2001) Beta-cell apoptosis: stimuli and signaling. Diabetes 50 Suppl 1, S58-S63

10. Kim WH, Lee JW, Suh $\mathrm{YH}$ et al (2005) Exposure to chronic high glucose induces $\beta$-cell apoptosis through decreased interaction of glucokinase with mitochondria. Diabetes 54, 2602-2611

11. Piro S, Anello M, Di Pietro C et al (2002) Chronic exposure 
to free fatty acids or high glucose induces apoptosis in rat pancreatic islets: possible role of oxidative stress. Metabolism $51,1340-1347$

12. Viswanathan SR, Daley GQ and Gregory RI (2008) Selective blockade of microRNA processing by Lin28. Science 320, 97-100

13. Viswanathan SR and Daley GQ (2010) Lin28a: a microRNA regulator with a macro role. Cell 140, 445-449

14. Newman MA, Thomson JM and Hammond SM (2008) Lin-28 interaction with the Let-7 precursor loop mediates regulated microRNA processing. RNA 47, 1539-1549

15. Zhu H, Shyh-Chang N, Segrè AV et al (2011) The Lin28/ let7 axis regulates glucose metabolism. Cell 147, 81-94

16. Yang DH and Moss EG (2003) Temporally regulated expression of Lin-28 in diverse tissues of the developing mouse. Gene Expr Patterns 3, 719-726

17. Piskounova E, Polytarchou C, Thornton JE et al (2001) Lin28A and Lin28B inhibit let-7 microRNA biogenesis by distinct mechanisms. Cell 147, 1066-1079

18. Frost RJ and Olson EN (2011) Control of glucose homeostasis and insulin sensitivity by the Let-7 family of microRNAs. Proc Natl Acad Sci U S A 108, 21075-21080

19. Balcazar Morales $N$ and Aguilar de Plata $C$ (2012) Role of AKT/mTORC1 pathway in pancreatic $\beta$-cell proliferation. Colomb Med 43, 235-243

20. Tuttle RL, Gill NS, Pugh W et al (2001) Regulation of pancreatic $\beta$-cell growth and survival by the serine/threonine protein kinase Akt1/PKB. Nature Med 10, 71133-71137

21. Kim DJ, Toda C, Ramírez CM et al (2017) Hypothalamic ventromedial Lin28a enhances glucose metabolism in diet-induced obesity. Diabetes 66, 2102-2111

22. Park KG, Lee KM, Seo HY et al (2007) Glucotoxicity in the INS-1 rat insulinoma cell line is mediated by the orphan nuclear receptor small heterodimer partner. Diabetes $56,431-437$
23. Kooptiwut S, Kaewin S and Semprasert N (2018) Estradiol prevents high glucose induced $\beta$-cell apoptosis by decreased BTG2 expression. Sci Rep 16, 12256

24. Chao DT and Korsmeyer SJ (1998) BCL-2 family: regulators of cell death. Annu Rev Immunol 16, 395-419

25. Mizuno N, Yoshitomi H, Ishida H et al (1998) Altered $\mathrm{BCL}-2$ and Bax expression and intracellular $\mathrm{Ca} 2+$ signaling in apoptosis of pancreatic cells and the impairment of glucose-induced insulin secretion. Endocrinology 139, 14291439

26. Brown JE and Dunmore SJ (2007) Leptin decreases apoptosis and alters BCL-2: Bax ratio in clonal rodent pancreatic beta-cells. Diabetes Metab Res Rev 23, 497-502

27. Wrede CE, Dickson LM, Lingohr MK et al (2002) Protein kinase B/Akt prevents fatty acid-induced apoptosis in pancreatic beta-cells (INS-1). J Biol Chem 277, 49676-49684

28. Wijesekara N, Krishnamurthy $M$, Bhattacharjee $A$ et al (2010) Adiponectin-induced ERK and Akt phosphorylation protects against pancreatic beta cell apoptosis and increases insulin gene expression and secretion. J Biol Chem 285, 33623-33631

29. Bernal-Mizrachi E, Wen W, Stahlhut S et al (2001) Islet beta cell expression of constitutively active Akt1/PKB alpha induces striking hypertrophy, hyperplasia, and hyperinsulinemia. J Clin Invest 108, 1631-1638

30. Sung Y, Jeong J, Kang RJ et al (2019) Lin28a expression protects against streptozotocin-induced $\beta$-cell destruction and prevents diabetes in mice. Cell Biochem Funct 37, 139-147

31. Zhang M, Niu X, Hu J et al (2014) Lin28a protects against hypoxia/reoxygenation induced cardiomyocytes apoptosis by alleviating mitochondrial dysfunction under high glucose/ high fat conditions. PLoS One 9, e110580

32. Jung GS, Hwang YJ, Choi JH et al (2020) Lin28a attenuates TGF- $\beta$-induced renal fibrosis. BMB Rep 53, 594-599 